\title{
Cortesía verbal y competencia lingüística: la petición cortés como tradición discursiva
}

\author{
VERBAL \\ POLITENESS \\ AND \\ LINGUISTIC \\ COMPETENCE:
}

POLITE REQUESTS AS DISCOURSE TRADITIONS

\author{
Angela Schrott \\ Universität Kassel
}

Resumen:

Muchas técnicas de atenuación lingüística están estrechamente ligadas a estrategias de cortesía verbal. Un clásico en este contexto son las preguntas del tipo ¿Puedes pasarme el pan, por favor? que funcionan como petición cortés. La cuestión central es cómo esta conocida técnica de la petición cortés puede ser situada en el sistema de la competencia lingüística según Eugenio Coseriu. Resulta preciso aclarar si se trata de tradiciones idiomáticas que pertenecen al saber lingüístico o si se trata de una tradición discursiva y cultural que se realiza en una o varias lenguas, pero que no forma parte de estas lenguas. En el campo de la atenuación lingüística y de la cortesía verbal esta diferenciación abre una perspectiva metodológica esclarecedora que permite describir de manera precisa la interacción entre el saber lingüístico y el saber cultural.

PALABRAS CLAVE: cortesía verbal, tradición discursiva, petición cortés, rutina comunicativa

Abstract:

Many techniques of linguistic mitigation are closely related to strategies of verbal politeness. A classic example are utterances of the type Could you please pass the bread? which function as a polite request. The core question is how this well-known communicative routine can be described in the interplay of language and culture. Based on Eugenio Coseriu's concept of the system of a language as a form of cultural competence we raise the question whether such routines belong to the linguistic traditions of a language or whether this technique is to be seen as a cultural discourse tradition that may be realized 
within various languages. In the field of linguistic mitigation and verbal politeness the concept of discourse tradition opens up a methodological perspective that enables us to describe the interaction of language and culture.

KEYWORDS: verbal politeness, discourse tradition, polite request, communicative routine

\section{EL MARCO TEÓRICO: LA LINGÜÍSTICA COMO CIENCIA CULTURAL}

Desde la perspectiva de la pragmalingüística la atenuación lingüística está estrechamente ligada a la cortesía verbal. El punto de partida de esta conexión es el hecho de que muchas estrategias de atenuación sirven para mitigar la fuerza ilocutiva de ciertos actos de habla (véase Mihatsch 2010: 47-51 para este concepto de la atenuación). En el contexto de la cortesía, el presente estudio analiza distintas estructuras interrogativas que funcionan como técnicas de la petición cortés. La cuestión central es cómo pueden ser situadas estas técnicas en una teoría de las tradiciones lingüísticas y culturales del habla partiendo del sistema de la competencia lingüística elaborado por Eugenio Coseriu.

La cortesía verbal es una forma de actuar en contextos culturales e históricos y por eso puede ser estudiada únicamente sobre la base de una lingüística orientada hacia la cultura. Tanto los fenómenos de la atenuación como la cortesía verbal son objeto de análisis de aquellas corrientes investigadoras que consideran a la lingüística una ciencia cultural (Gardt 2003: 272 y 2012: 294-296; Schrott 2015: 115-119). En el ámbito de la romanística, la orientación hacia una lingüística cultural gira en torno a varias líneas tradicionales. El enlace teórico más importante entre idioma y cultura - más allá de lo histórico - hay que buscarlo en el sistema coseriano de la competencia lingüística. Me refiero al conocido modelo de los tres niveles de la competencia lingüística postulado por Coseriu, la cual es definida expresamente como una competencia cultural. Esta definición se funda en el hecho de que el hablar, siendo energeia, da lugar a tradiciones y, de esta manera, crea ámbitos de cultura (Coseriu 1992: 81, 86-87). Ya que el hablar, así entendido, constituye un acto cultural, los saberes que confluyen en el habla han de ser definidos como componentes de una competencia lingüística cultural. De ahí que quiera proponer un modelo pragmalingüístico como base teórica, el cual parte del sistema coseriano. Como es sabido, la pragmática lingüística se centró primero en posibles categorías universales, como los actos de habla universales (Searle 1969, 1979a) y los principios y máximas generales del hablar (Grice 1989). A esto se añadieron como contrapeso importante corrientes como la pragmática histórica y la pragmática contrastiva, desviando la atención desde los universales lingüísticos hacia las diferencias lingüísticas y culturales. De esta perspectiva se derivan dos desafíos para la pragmática contrastiva (véase Trosborg 2010: 911, 28-31). Por un lado, se trata de restituir diferencias culturales y lingüísticas a los valores universales: el estudio de lo universal comprende también el estudio de lo particular. Por otro 
lado, se trata de describir y explicar el enlace que existe entre lengua y cultura en sus interacciones concretas.

\section{UN MODELO DE LA PRAGMÁTICA LINGÜÍSTICA: REGLAS Y TRADICIONES}

Según Coseriu (1992: 86) el hablar es una actividad humana universal que se manifiesta en las lenguas históricas particulares, y que siempre es realizada por los hablantes individuales en situaciones comunicativas determinadas. A estas tres características corresponden tres planos del hablar, contando cada uno con un tipo de saber específico.

\begin{tabular}{ccc}
\hline $\begin{array}{c}\text { plano } \\
\text { universal }\end{array}$ & $\begin{array}{c}\text { reglas y principios } \\
\text { generales }\end{array}$ & saber universal \\
\hline plano histórico & $\begin{array}{c}\text { tradiciones } \\
\text { idiomáticas }\end{array}$ & saber lingüístico \\
\hline plano & tradiciones & saber cultural para realizar \\
individual & discursivas & tareas comunicativas \\
\hline
\end{tabular}

Esquema 1: Reglas y tradiciones del hablar según Eugenio Coseriu 1992

El plano universal contiene reglas y principios generales y universales del hablar. Son independientes de la evolución histórica y se aplican al hablar en todas las lenguas y culturas. No forman parte del saber lingüístico y, por tanto, no son exclusivos de ningún idioma (Coseriu 1992: 90-91, 180-181, 183-186). El plano histórico de las lenguas particulares comprende el saber idiomático que hace posible el hablar en distintas lenguas. Las tradiciones idiomáticas engloban la sintaxis, el léxico, la fraseología: es decir, todo el sistema de la lengua que permite al hablante comunicarse en una lengua particular, sea el español, el alemán u otra. Dado que este saber se transmite de generación en generación hablo aquí de "tradiciones idiomáticas". El plano individual de los textos y de los discursos comprende las tradiciones discursivas. Representan un saber cultural que guía a los hablantes en la correcta realización de sus tareas comunicativas. Las tradiciones discursivas comprenden rutinas comunicativas como, por ejemplo, los saludos o la formulación de una invitación; también pueden ser más complejas y servir como instrucciones para una ponencia científica, para un texto periodístico o para una novela. ${ }^{1}$ Tanto las tradiciones idiomáticas como las tradiciones discursivas son aprendidas y transmitidas por los hablantes como tradiciones. No obstante, las dos tradicionalidades son sustancialmente diferentes. Las tradiciones idiomáticas forman un saber lingüístico que caracteriza a un determinado idioma. Las tradiciones discursivas, en cambio, constituyen un

\footnotetext{
${ }^{1}$ Coseriu 1992: 92 utiliza términos diferentes para denominar los tres tipos de saber (saber elocutivo, idiomático y expresivo). La terminología aquí elegida representa la diferencia entre reglas generales universales y tradiciones históricas del hablar. Véase Schlieben-Lange 1983: 138-140, Koch 1997: 45, 47-49 y 2008: 53-65, Oesterreicher 2001: 1558-1559, Lebsanft 2005: 30-31 y 2015: 100-104.
} 
saber cultural que se puede practicar en varios idiomas, o que solamente está ejercido por una parte de los hablantes dentro de una comunidad lingüística.

Como en cada texto influyen tanto tradiciones idiomáticas como tradiciones discursivas, los textos siempre forman parte de dos tradicionalidades. El hecho de que el modelo de la competencia lingüística parta fundamentalmente del hablar como actividad (energeia) es el punto de partida para mi reinterpretación, la cual se orienta hacia un modelo de la pragmática lingüística (véase Schrott 2014: 9-12, 2015: 120-123):

\begin{tabular}{|c|c|c|c|}
\hline $\begin{array}{l}\text { reglas y } \\
\text { tradiciones }\end{array}$ & $\begin{array}{c}\text { reglas y principios } \\
\text { generales }\end{array}$ & $\begin{array}{l}\text { tradiciones } \\
\text { idiomáticas }\end{array}$ & $\begin{array}{l}\text { tradiciones } \\
\text { discursivas }\end{array}$ \\
\hline $\begin{array}{l}\text { ámbitos de la } \\
\text { pragmática }\end{array}$ & $\begin{array}{c}\text { pragmática } \\
\text { general }\end{array}$ & $\begin{array}{l}\text { pragmática de la } \\
\text { lengua particular }\end{array}$ & $\begin{array}{l}\text { pragmática de } \\
\text { las tradiciones }\end{array}$ \\
\hline perspectivas & $\begin{array}{c}\text { perspectiva } \\
\text { universal }\end{array}$ & $\begin{array}{l}\text { perspectiva de la } \\
\text { lengua particular }\end{array}$ & $\begin{array}{l}\text { perspectiva } \\
\text { cultural }\end{array}$ \\
\hline
\end{tabular}

Esquema 2: Las tres perspectivas de la pragmática lingüística

Partiendo de los tres tipos de saber se derivan - según la focalización - tres ámbitos y tres perspectivas de la pragmática. ${ }^{2}$ La pragmática general y universal se dedica a las reglas generales del hablar, como el principio de la cooperación y sus máximas, para determinar lo que es universal en el habla. La pragmática de las lenguas particulares estudia las estructuras lingüísticas primariamente desde una perspectiva pragmático-funcional; un análisis de los marcadores del discurso en español pertenecería a este ámbito de la pragmática que se centra sobre todo en las estructuras lingüísticas de una determinada lengua. La pragmática de las tradiciones discursivas investiga las tradiciones y normas culturales del habla en contextos concretos, es decir que se dedica a analizar el saber cultural que es el hilo conductor para cumplir diferentes tareas comunicativas en el uso de la lengua. Por eso, la pragmática de las tradiciones discursivas no se concentra necesariamente en una sola lengua sino que analiza una estrategia del habla en diferentes lenguas.

Los tres ámbitos de la pragmática lingüística expuestas aquí contienen tres perspectivas diferentes sobre el lenguaje y el habla. Como punto de partida sirve la definición de la pragmática lingüística como disciplina que interpreta el lenguaje y las estructuras lingüísticas

\footnotetext{
${ }^{2}$ Para la pragmalingüística como perspectiva véase Verschueren 1995: 11 y 2009: 14-18, Gardt 1999: 353, Fetzer 2011: 25-31.
} 
en los entornos y contextos de su uso (Verschueren 1995: 11 y 2009: 14-18, Gardt 1999: 353, Fetzer 2011: 25-31). Partiendo del esquema 2 se pueden diferenciar tres perspectivas pragmáticas. La pragmática general universal aborda el habla humana desde una perspectiva universal, y así se enfrenta a dos puntos de vista con orientación histórica: la historicidad de las lenguas particulares por un lado y la historicidad de las tradiciones discursivas por otro.

La ventaja esencial de este modelo de la pragmalingüístia, basado en el esquema coseriano, es precisamente que aborda la diferenciación entre el saber lingüístico y el saber cultural, lo cual facilita enormemente una clasificación entrecruzada - universal vs. histórico, lingüístico vs. idiomático. La referencia a Coseriu permite arrojar luz a la interacción entre lengua y cultura mediante la clara distinción entre las tradiciones idiomáticas como saber lingüístico - el sistema de la lengua - y las tradiciones discursivas como saber cultural. Así, el sistema de Coseriu facilita a la pragmática lingüística un sistema que permite diferenciar los componentes lingüísticos y culturales del hablar y distinguir exactamente, gracias a esta diferenciación, la interdependencia de las dos tradicionalidades.

\section{LA PETICIÓN CORTÉS Y EL CONCEPTO DE LA TRADICIÓN DISCURSIVA}

\subsection{La pregunta como petición cortés: su mecanismo pragmático}

En el marco de nuestro modelo de pragmática lingüística se analizará, a lo largo de los siguientes párrafos, la petición cortés como ejemplo "clásico" de la pragmática lingüística, que conecta el tema de la atenuación con el de la cortesía. ${ }^{3}$ El funcionamiento de la petición cortés se explica por el distinto perfil ilocutivo que tienen los actos interrogativos - o preguntas - y los actos volitivos:

(1) ¿Dónde es la conferencia plenaria?

(2) Dígame dónde es la conferencia plenaria.

Como es sabido, las preguntas como ¿Dónde es la conferencia plenaria? (1) destacan una falta de información por parte del hablante y apelan al destinatario a remediar este déficit indicando el lugar de la conferencia. No obstante, el acto deseado no se expresa explícitamente, sino que existe la expectativa de que el destinatario infiera, del déficit de información indicado, el deseo del hablante, y que cumpla con este deseo de manera cooperadora (Kerbrat-Orecchioni 2001: 84-86). Al fin y al cabo, es responsabilidad del destinatario extraer la inferencia adecuada y suministrar la información restante. Los actos interrogativos son, por ende, actos de habla, cuyo potencial activador resulta de la tematización de un déficit informativo; sin embargo, ellos no formulan de manera explícita la reacción deseada en la contestación (Escandell Vidal 1999: 3932-3933, Schrott 2014: 13-16, 18-19). Esta diferencia en lo que a la explicitud se refiere, distingue el ejemplo (1) del ejemplo

\footnotetext{
${ }^{3}$ Las peticiones son uno de los actos ilocutivos más estudiados. Véase por ejemplo Marquéz Reiter 2000, Placencia y Bravo, coord., 2002, Bravo y Briz, coord., 2004, Schrader-Kniffki, coord., 2006, Placencia y García, coord., 2007. Resúmenes todavía actuales del "state of the art" se encuentran en Placencia y García 2007a y 2007b.
} 
(2). A diferencia del acto interrogativo en (1), el acto volitivo (2) expresa explícitamente la acción esperada, denominada de manera explícita con el verbum dicendi. ${ }^{4}$ De un modo general, tanto las preguntas como los actos volitivos son altamente apelativos; no obstante, la activación del destinatario se realiza de manera diferente.

En la pragmática lingüística esta diferenciación ha sido discutida desde posiciones de signo contrario. Siguiendo la tradición de Searle (1969: 66-67) los actos interrogativos se interpretan como un subtipo de la volición que pide la transmisión de informaciones. ${ }^{5}$ Sin embargo, esta categorización no toma en consideración una diferencia importante entre preguntas y actos volitivos: mientras que la volición formula la acción deseada explícitamente - decir dónde es la conferencia -, el acto interrogativo renuncia a denominar la acción del decir y funciona como una invitación al interlocutor a inferir la acción relevante.

Esta diferencia en el perfil ilocutivo es importante para el análisis de actos interrogativos que funcionan como voliciones atenuadas y corteses. En estas locuciones una pregunta surte el efecto de una petición cortés y se presenta como una volición atenuada:

(3) ¿Puedes pasarme el pan, por favor?

(4) Tu pourrais me passer le pain, s'il te plaît?

(5) Could you please pass the bread?

(6) Kannst du mir bitte das Brot herübergeben?

Los ejemplos en español francés, inglés y alemán - se trata de la misma petición, expresada en diferentes lenguas - ilustran un clásico de la pragmática lingüística y de los estudios sobre la cortesía verbal (Briz 2004: 76, Blum-Kulka 1987: 141, 144, 1989: 41-43). Se trata de una estrategia cortés altamente convencionalizada en muchas lenguas, aunque no en todas (véase Wierzbicka 2003: 203-204, Schrott 2014: 14-16). ${ }^{6}$

Según Escandell Vidal (1988: 559-563, 1999: 3975-3978) esta técnica del acto volitivo realizado por la pregunta funciona como "interrogación directiva". Es decir, que un acto interrogativo sustituye a un acto volitivo con el fin de conseguir un efecto atenuador. El resultado es - según esta perspectiva - un interrogante apelativamente fuerte o un enunciado atenuado en su fuerza apelativa que se percibe como requerimiento cortés. Las interrogaciones directivas funcionan como rutinas comunicativas (Coulmas 1981: 13) que están conectadas gracias a una convención con contextos y marcos conceptuales semánticos de las peticiones corteses.

\footnotetext{
${ }^{4}$ Sobre la delimitación entre acto interrogativo y acto volitivo véase Schrott 2014: 14-16, Waldenfels 1994: 14, 55, 59, Bucher 1994: 241-242.

"Searle define los actos interrogativos como "requests for information". Basándose en esta definición, según Searle la locución

"Tell me the name of the first President of the United States" equivale a "What's the name of the first President of the United States?" (1969: 66-67, 69).

${ }^{6}$ Para el español véase Escandell Vidal (1988: 559-560, 1999: 3975-3978, Díaz Pérez 2003: 260), para el francés Mulken (1996: 698-699) y Kerbrat-Orecchioni (2001: 33-52, 85) y para el inglés Brown y Levinson (1987: 132-134).
} 
Queda claro que la selección de una u otra estructura idiomática para realizar una petición sea la interrogación directiva, sea un imperativo - no depende exclusivamente de la comunidad cultural sino también del contexto social - la relación entre los interlocutores y el nivel de imposición que suponga lo pedido - y de la distancia o inmediatez comunicativa que caracteriza la situación. En lo que ahora sigue el enfoque no está en un estudio comparativo de diferentes rutinas comunicativas de la petición o volición sino en la "interrogación directiva" y su estatus dentro de la competencia lingüística.

Los enunciados citados (3), (4), (5) y (6) tienen en común el hecho de que todos ellos están expresados por oraciones interrogativas y, por tanto ,muestran aquella estructura sintáctica que más conectada está con el acto interrogativo. Estas locuciones solamente funcionan como volición cortés si están expresadas a través de la estructura de las frases interrogativas. Obsérvese que una reformulación del tipo "Yo te pregunto si puedes pasarme el pan" no puede expresar una volición cortés. Además, los cuatro ejemplos aluden en la proposición de la pregunta a la capacidad y la disposición del destinatario. Por tanto, literalmente se pregunta por la existencia de una capacidad: (3) puedes, (4) tu pourrais (5) could you (6) kannst du. Mientras la estructura interrogativa a nivel sintáctico es obligatoria para el funcionamiento de la petición cortés, la tematización de la capacidad a nivel semántico es frecuente pero no obligatoria, como lo demuestran ejemplos como ¿Me pasas el pan, por favor?.

El fenómeno de que un acto interrogativo sea percibido como una volición cortés, es explicado en la lingüística pragmática muchas veces como un acto de habla indirecto - según la diferenciación de Searle - en el cual el hablante realiza una volición por medio de un acto interrogativo. Esto significa que en los actos de habla indirectos confluyen dos actos de habla realizados a la vez cuando se ha de inferir el acto de habla propiamente indicado. ${ }^{7}$ No obstante, suponer dos actos de habla diferentes, realizados simultáneamente, es problemático, ya que el acto interrogativo y la volición, en el caso de las interrogaciones directivas, tienen un estatus diferente. La volición es, de hecho, una inferencia del destinatario, y no un acto de habla llevado a cabo por el hablante. Otro argumento para postular solamente un único acto de habla es el hecho de que esta técnica sea aplicada exclusivamente a oraciones interrogativas y, por lo tanto, haga uso de aquella estructura que más conectada está con los actos interrogativos.

Partiendo de esa base, quisiera explicar el funcionamiento de la interrogación directiva como técnica de la atenuación apelativa. El enunciado ¿Puedes pasarme el pan, por favor? funciona como acto interrogativo que confiere al destinatario la opción entre una respuesta positiva (Sí, puedo pasarte el pan) o una respuesta negativa (No, no puedo pasarte el pan). En la gran mayoría de los casos se trata de una opcionalidad fingida que no ofrece una verdadera libertad al destinatario. No obstante, el acto interrogativo funciona como una invitación para realizar una inferencia. En el caso normal el destinatario realizará la inferencia como se esperaba y

\footnotetext{
${ }^{7}$ Sobre el "indirect speech act" véase Searle 1979b: 30-31, 43-44. Sobre la discusión sobre los actos de habla indirectos véase Meibauer 1986: 32-42, Haverkate 1994: 161-163, Lempert 2012: 182-185, 189-190 y Wierzbicka 2010:46-48.
} 
llevará a cabo la acción deseada. Sin embargo, al menos en casos singulares tiene la posibilidad de no llevar a cabo ninguna inferencia y resistirse así al deseo del hablante. ${ }^{8}$

Dicha opcionalidad no existe en el caso de los actos volitivos como Pásame el pan, ya que se le asigna al destinatario explícitamente el papel del agente ejecutivo y si se negara a reaccionar, ello significaría siempre rechazar explícitamente la demanda. Mientras que el acto interrogativo le asigna al destinatario el papel de un interlocutor cooperativo que sabe sacar las conclusiones adecuadas del déficit de información señalado y que, además, sabe elegir la opción adecuada y cooperativa, el acto volitivo le asigna al destinatario el papel de un actante que ejerce la voluntad ajena. La tematización diferente del acto deseado, la existencia de una opcionalidad y los papeles diferentes de los destinatarios son razones de peso para considerar que las voliciones son claramente más insistentes que las preguntas. Todos estos factores justifican una clara separación entre preguntas y voliciones. Mientras que los actos volitivos presuponen una predisposición a actuar, los actos interrogativos no precisan de ello.

La atenuación de carácter apelativo así producida se puede explicar a través del concepto del face, un concepto de gran influencia en la investigación sobre la cortesía verbal. Según Brown y Levinson (1987: 61.63, 65-74) el face contiene dos componentes como imagen propia socialmente facilitada: "positive face" y "negative face". La "imagen positiva", consiste en la aspiración a adquirir la estimación de los demás, mientras la "imagen negativa" consiste en la necesidad de actuar de manera autodeterminante. En consecuencia, los actos volitivos están considerados sobre todo como potenciales "face threatening acts"; sin embargo, aquellos actos de habla que permiten opciones reducen este "riesgo", ya que conceden al destinatario una libertad de decisión (real o fingida) sobre la acción tematizada. Dado que los actos interrogativos, por lo menos teóricamente, ofrecen una opcionalidad al interlocutor, funcionan como técnicas de la cortesía negativa, la cual protege la autonomía del interlocutor y sirve a menudo para atenuar actos directivos (Brown y Levinson 1987: 72-74, 102, 130-132). Sin embargo, al mismo tiempo, dejar opciones también favorece la imagen positiva, ya que un hablante, al prescindir de voliciones explícitas, señala que considera al otro como interlocutor cooperador y que le otorga así confianza comunicativa. En resumen, dar opciones es una estrategia que satisface tanto la imagen positiva como la imagen negativa.

Ya que ofrecer opciones le deja al interlocutor la libertad de elegir, esta estrategia tiene un gran valor interaccional, sobre todo en sociedades en las que las libertades individuales son importantes. Sin embargo, esta tendencia no es universal, ya que también existen comunidades en las que se prefieren técnicas de franqueza como manifestación de cordialidad, porque estas técnicas están conectadas con una conducta afectiva altamente apreciada. Un ejemplo sería el grado de insistencia con el cual se puede formular una invitación. Mientras en ciertas culturas una invitación o una oferta puede formularse con gran

\footnotetext{
${ }^{8}$ Para el concepto de opcionalidad véase Lakoff 1973: 298, Leech 1983: 108, 132, Díaz Pérez 2003: 253-254 y Siebold 2008: 104-106.
} 
énfasis - "Tienes que probar otro pastelito, toma, toma" - otras comunidades culturales entenderían tal enunciado como una transgresión que lesiona la autonomía del otro (Wierzbicka 2010: 46-48).

\subsection{La tradicionalidad idiomática y la tradicionalidad cultural}

La facticidad reducida y la opcionalidad transmitidas por medio de los actos interrogativos sirven como medio de atenuación. Con referencia al modelo de pragmalingüística expuesto en el capítulo 2, resulta preciso aclarar si se trata de una regla universal, de una tradición idiomática, que pertenece al saber lingüístico, o de una tradición discursiva como saber cultural. Esta diferenciación es importante porque abre una perspectiva metodológica esclarecedora hacia los patrones ilocucionarios y las rutinas comunicativas de atenuación que permite describir de manera más precisa el funcionamiento de las estrategias de cortesía entre el saber lingüístico y el saber cultural.

La pragmática contrastiva demostró que el patrón ¿Puedes pasarme el pan, por favor? existe como estrategia cortés altamente convencionalizada en muchas lenguas, como por ejemplo en español (Escandell Vidal 1999: 3975-3978, Díaz Pérez 2003: 260), en francés (Mulken 1996: 698-699, Kerbrat-Orecchioni 2001: 33-52, 85) o en inglés (Brown y Levinson 1987: 132134). Sin embargo, no se trata de una estrategia universal. Según Wierzbicka (2003: 32-37) peticiones realizadas mediante un acto interrogativo no se usan con alta frecuencia en polaco y los hablantes nativos tienen que aprender que este tipo de pregunta funciona como "pragmatic idiom" en inglés o en español (Ogiermann 2012: 43-45). Por eso, la estrategia de realizar una petición usando un acto interrogativo es un patrón dependiente de la cultura y, por tanto, no forma parte de las reglas universales del hablar - a pesar de su difusión en muchas comunidades lingüísticas y áreas culturales (véase Wierzbicka 2003: 203-204, Schrott 2014 : 14-16).

Ahora bien, queda por aclarar si en el caso de las peticiones atenuadas se trata de tradiciones idiomáticas de fijación parcialmente formulaica o si se trata de una tradición discursiva y cultural que se realiza en una o varias lenguas, pero que no forma parte de las tradiciones idiomáticas de estas lenguas. La petición cortés del tipo “¿Puedes pasarme el pan?” es un procedimiento que emplea de manera obligatoria estructuras interrogativas. Por tanto, está ligado con determinados tipos de oraciones, los cuales - para seguir en el marco del esbozo de los tres tipos del saber - forman tradiciones idiomáticas del alemán, del español, del francés y del inglés. La interrogación directiva recurre a estas estructuras de las lenguas particulares y se manifiesta a través del material lingüístico a nivel del idioma particular.

Sin embargo, ella misma no forma parte de la lengua particular sino que es una tradición cultural cuyo radio de acción no coincide con las fronteras lingüísticas. Con respecto a nuestro modelo de la pragmática lingüística se puede deducir que la interrogación directiva no es una tradición idiomática, sino más bien una tradición cultural del habla determinada por la cultura. Se trata de una tradición discursiva que utiliza estructuras idiomáticas específicas. Siendo una 
tradición discursiva de la volición cortés, mueve a los interactuantes a elegir, dentro del repertorio del idioma particular, la frase interrogativa más adecuada como recurso para realizar una petición cortés. Desde esta perspectiva, las estructuras interrogativas son elementos elegidos por la tradición discursiva.

\subsection{La tradición discursiva de la petición cortés en diferentes lenguas}

La tradición discursiva de la interrogación directiva comprende en las distintas lenguas un abanico más o menos largo de variantes: algunas lenguas particulares tienen un abanico más grande que otras. Además de las interrogaciones totales, también las interrogaciones parciales pueden funcionar como interrogaciones directivas. ${ }^{9}$ Los enunciados (7) a (10) son el resultado de una encuesta entre hablantes nativos. El punto de partida es la situación comunicativa siguiente: dos colegas están en una reunión de trabajo; uno de ambos tiene pasteles para una merienda y dice a su colega:

\footnotetext{
(7) Why don't you get us some more coffee?

(8) ¿Por qué no nos preparas un poco de café?

(9) Pourquoi tu ne nous prépares pas un petit café?

(10) Warum machst du uns nicht einen Kaffee?
}

La interrogación parcial negativa en inglés (7), introducida con why, en este caso no pregunta por la razón por la cual una determinada acción hasta ahora no ha sido (o es) llevada a cabo. No se trata de cubrir un déficit de información, sino de una volición cortés del hablante para pedirle algo al destinatario (Jung/Schrott 2003: 249-250, House 1997: 82-83). En inglés se usa la tradición idiomática de la interrogación parcial para sugerirle al destinatario la inferencia de que no hay ninguna razón para negarse a realizar la acción indicada en la proposición. Enunciados del tipo Why don't you get us some more coffee? constituyen una rutina conversacional altamente convencionalizada en inglés y funcionan como tradición discursiva de la petición cortés. Este tipo de interrogación se usa también como petición cortés en español (8), aunque el valor de petición parece menos convencionalizado que en inglés: se le cede al destinatario la posibilidad de reconocer que no hay impedimento para realizar la acción, lo cual le invita a realizar la acción, cumpliendo así con el deseo del hablante (Monjour 2006: 25-27, Mulder 2003: 181). Sin embargo, la interrogación negativa, preguntando por la causa o la razón, no se usa como petición cortés convencionalizada en francés (9) o en alemán (10). Es más, las versiones del francés (9) y del alemán (10) se suelen entender como verdaderas preguntas de un locutor que quiere saber la razón por la cual el otro no quiere preparar café.

Al comparar el inglés, el español, el francés y el alemán se puede concluir que la interrogación directiva realizada por una interrogativa total del tipo ¿Puedes pasarme el pan? existe en los cuatro idiomas, como lo muestran los ejemplos (3) a (6). Sin embargo, la interrogación directiva realizada por una interrogativa parcial negativa, que tiene en inglés y en español el estatus de

\footnotetext{
${ }^{9}$ Mientras la interrogación total (¿Vamos al cine?) exige una respuesta afirmativa o negativa - sí o no - , la interrogación parcial pregunta por un determinado elemento oracional (¿Cuando vamos al cine?). Véase Alcina Franch/Blecua 1998: 925.
} 
una rutina comunicativa, está en cambio mucho menos establecida como fórmula rutinaria en alemán y en francés. Todos los idiomas aquí mencionados disponen de la tradición idiomática de la interrogativa parcial, sin embargo solamente se elige la interrogación parcial negativa como tradición discursiva de la petición cortés en la lengua y cultura anglófona y en el mundo hispanohablante. ${ }^{10}$

Partiendo de una competencia lingüística entendida como competencia cultural se plantea la cuestión de cómo se puede explicar esta diferencia al nivel de las tradiciones discursivas. Una posible explicación es que la tradición discursiva de la petición cortés tiene a su disposición una diferente gama de estructuras en diferentes lenguas. En el caso del inglés la interrogación total y la interrogación parcial pertenecen a la tradición discursiva de la interrogación directiva mientras que en alemán o en francés la tradición discursiva de la petición cortés se realiza exclusivamente por medio de la interrogación total. Así pues, se puede concluir que diferentes idiomas usan la misma tradición discursiva de la interrogación directiva, la cual, sin embargo, está realizada en los diferentes idiomas con una complejidad distinta.

\section{LA PETICIÓN CORTÉS Y LAS REGLAS UNIVERSALES DEL HABLA: EL PRINCIPIO DE LA COOPERACIÓN}

El sistema de Eugenio Coseriu, con su clara distinción entre las tradiciones idiomáticas y las tradiciones discursivas, permite identificar la interrogación directiva como un saber cultural y una tradición discursiva. Mientras que mis precedentes consideraciones se concentraron en las tradicionalidades idiomática y discursiva querría volver ahora al plano universal del sistema coseriano. Una de las reglas universales en este plano - quizás la más importante - es el principio de cooperación según Grice (Grice 1989: 26-28, Lebsanft 2005: 26-27, 32). En las investigaciones sobre la cortesía verbal muchas veces es subrayado el hecho de que hablar cortésmente a menudo contradice las máximas del principio cooperador o, por lo menos, eso es lo que parece. Esto también se puede aplicar a los procedimientos de la atenuación.

Visto el asunto desde la perspectiva de las máximas de Grice se puede considerar la interrogación directiva como una técnica que incumple la máxima de relevancia (Maxim of Relation), así como la máxima de pertinencia (Maxim of Manner), centrada en la claridad (perspicuitas) del habla. Actos directivos, como por ejemplo Pásame el pan, por favor, verbalizan inequívocamente la voluntad del hablante relevante en la situación comunicativa. Además, no reclaman ninguna inferencia por parte del destinatario y gozan pues como actos de habla de una gran claridad y relevancia. Pero a diferencia del acto directivo, que se realiza muchas veces a través de un imperativo, la interrogación directiva (¿Puedes pasarme el pan, por favor?) requiere llevar a cabo una inferencia (aunque sea convencionalizada). Fundamentalmente, hay dos maneras de percibirla: como petición convencionalizada o como pregunta relativa a la capacidad, con lo cual posee una ambigüedad fundamental, aunque en

\footnotetext{
${ }^{10}$ Este tipo de la interrogativa parcial se usa como petición atenuada no solo en el español peninsular, sino también en diversas variedades hispanoamericanas, véase por ejemplo Marquéz Reiter 2000, Placencia y Bravo, coord., 2002, Placencia y García, coord., 2007.
}

Normas (ISSN: 2174-7245) | 
la mayoría de los casos elegir la manera adecuada de percibirla no constituye ningún problema. Desde este punto de vista, un requerimiento del tipo Coge el teléfono parece ser más relevante y más evidente que el enunciado ¿Por qué no coges el teléfono?, que funciona como interrogación directiva (Mulder 2003: 181). Debido a su opcionalidad, las interrogaciones directivas dan la impresión de realizar las máximas de relevancia y de claridad solo parcialmente, o incluso socavarlas indicando simplemente la voluntad del hablante sin formular explícitamente esta volición.

No obstante, se puede conectar la interrogación directa de dos maneras diferentes al principio de cooperación: de manera interna y de manera externa. Por un lado, se puede entender el principio de cooperación como un valor que se puede ampliar de manera externa por reglas adicionales, como el principio de la cortesía. Desde esta perspectiva, hablar de manera cortés parece ser un 'valor agregado' que añade un interactuante al principio cooperativo, y dejar una opción se puede interpretar como 'valor agregado' externo que complementa el principio cooperativo (Grice 1989: 28-30, Leech 1983: 10, 81-83). Por otro lado, se puede explicar la cortesía verbal y la opcionalidad de manera interna, dentro del principio de cooperación. En el caso de la interrogación directiva se indica claramente la inferencia a través de la correlación formal con la frase interrogativa, por lo cual funciona como convención conocida. Con referencia a las Máximas de Grice, las interrogaciones directivas como estrategias de la atenuación no son necesariamente menos evidentes o menos relevantes que los requerimientos, ya que en una cultura conversacional de la opcionalidad son fáciles de identificar.

Además hay que tener en cuenta que la relevancia y la claridad son valores sometidos a modificaciones culturales: una volición valorada por su claridad ejemplar en una comunidad cultural puede ser considerada como demasiado "clara" y poco cortés en otra cultura. También la evaluación de interrogaciones directivas, sobre la base de las máximas de Grice, depende mucho del tipo ideal del interlocutor cooperador cultivado por una sociedad. La relevancia 'disminuida' y la claridad de las interrogaciones directas cuentan con el destinatario que reconoce y estima que le dejen opciones, y así le dan al interlocutor un anticipo de confianza comunicativa. La conexión con la máxima de relevancia aquí esbozada indica que las técnicas de la atenuación o de la cortesía no forman un sistema aislado de normas, sino que pueden ser vinculadas al cooperative principle y sus máximas.

\section{BIBLIOGRAFÍA}

Alcina Franch, Juan/BlecuA, José Manuel (1998): Gramática española. Ariel, Barcelona.

BLUM-KULKA, Shoshana (1987): «Indirectness and politeness in requests: same or different?", Journal of Pragmatics, 11, 131-146.

BLUM-KULKA, Shoshana (1989): "Playing it Safe: The Role of Conventionality in Indirectness", en BlumKulka, Shoshana et alii, coord., Cross-Cultural
Pragmatics: Requests and Apologies, Norwood, Ablex, 37-70.

Bravo, Diana y Antonio BRIZ, coord., (2004): Pragmática sociocultural: estudios sobre el discurso de cortesía en español, Barcelona, Ariel, 67-94.

BRIZ, Antonio (2004): «Cortesía verbal codificada y cortesía verbal interpretada en la conversación», 
en Bravo, Diana y Antonio Briz, coord., Pragmática sociocultural: estudios sobre el discurso de cortesía en español, Barcelona, Ariel, 67-94.

Brown, Penelope y Stephen C. LeVINSON (1987): Politeness. Some Universals in Language Use, Cambridge y otras, Cambridge University Press.

BUCHER, Hans-Jürgen (1994): «Frage-AntwortDialoge», en Hundsnurscher, Franz y Gerd Fritz, coord., Handbuch der Dialoganalyse, Tübingen, Niemeyer, 239-258.

COSERIU. Eugenio (1992): Competencia lingüística. Elementos de la teoría del hablar, Madrid, Gredos.

COULMAS, Florian (1981): Routine im Gespräch. Zur pragmatischen Fundierung der Idiomatik, Wiesbaden, Akademische Verlagsgesellschaft Athenaion.

DíAz PÉREZ, Francisco J. (2003): La cortesía verbal en inglés y en español. Actos de habla y pragmática intercultural, Jaén, Universidad de Jaén.

ESCANDELL VIDAL, María Victoria (1988): La interrogación en español. Semántica y pragmática, Madrid, Universidad Complutense.

ESCANDELL VIDAL, María Victoria (1999): «Los enunciados interrogativos: aspectos semánticos y pragmaticos», en Bosque, Ignacio y Violeta Demonte, coord., Gramática descriptiva de la lengua española, tomo 3, Madrid, Espasa Calpe, 3929-3992.

FETZER, Anita (2011): «Pragmatics as a linguistic concept», en Bublitz, Wolfram y Neal R. Norrick, coord., Foundations of Pragmatics, Berlin/New York, De Gruyter, 23-50.

GARDT, Andreas (1999): Geschichte der Sprachwissenschaft in Deutschland. Vom Mittelalter bis ins 20. Jahrhundert, Berlin/New York, De Gruyter.

GARDT, Andreas (2003): "Sprachwissenschaft als Kulturwissenschaft», en Haß-Zumkehr, Ulrike y Christoph König, coord., Literaturwissenschaft und Linguistik von 1960 bis heute, Göttingen, Wallstein Verlag, 271-288.

GARDT, Andreas (2012): «Sprachgeschichte als Kulturgeschichte», en: Péter Maitz, coord., Historische Sprachwissenschaft. Erkenntnisinteressen, Grundlagenprobleme, Desiderate, Berlin/Boston, 289-300.

GofFMAN, Erving (1967): "On Face-Work. An Analysis of Ritual Elements in Social Interaction», en Goffmann, Erving, Interaction Ritual: Essays on
Face-to-Face Behavior, New York, Pantheon Books, 5-45.

GRICE, H. Paul (1989): «Logic and conversation», en Grice, H. Paul, Studies in the Way of Words, Cambridge, Mass./London, Harvard University Press, 22-40 (prim. en Cole, Peter y Jerry L. Morgan, coord., Syntax and Semantics, tomo 3. Speech Acts, New York, Academic Press, 4158).

HAVERKATE, Henk (1994): La cortesia verbal. Estudio pragmalingüístico, Madrid, Gredos.

HouSE, Juliane (1997): Translation Quality Asssesssemnt - A Model Revisited. Tübingen: Narr.

JUNG, Verena y Angela SCHROTT (2003): «A Question of Time? Questions types and speech act shifts from a historica-contrastive perspective», en: Kaszia Jazszolt y Ken Turner, coord., Meaning Through Language Contrast, Amsterdam/Philadelphia, Benjamis, 345-371.

KERBRAT-ORECCHIONI, Catherine (2001): Les actes de langage dans le discours. Théorie et fonctionnement, Paris, Nathan.

KoCH, Peter (1997): "Diskurstraditionen: zu ihrem sprachtheoretischen Status und ihrer Dynamik», en Frank, Birgit et alii, coord., Gattungen mittelalterlicher Schriftlichkeit, Tübingen, Narr, 43-79.

KoCH, Peter (2005): «Sprachwandel und Sprachvariation", en Schrott, Angela y Harald Völker, coord., Historische Pragmatik und historische Varietätenlinguistik in den romanischen Sprachen, Göttingen, Göttinger Universitätsverlag, 229-254.

$\mathrm{KoCH}$, Peter (2008): "Tradiciones discursivas y cambio lingüístico: el ejemplo del tratamiento vuestra merced en español», en Kabatek, Johannes, coord., Sintaxis histórica del español y cambio lingüístico: Nuevas perspectivas desde las tradiciones discursivas, Frankfurt am Main/Madrid, Vervuert, 53-87.

LAKOFF, Robin (1973): «The Logic of Politeness; or: Minding your P's and Q's», CLS 9: Papers from the ninth regional meeting of the Chicago linguistic society, 292-305.

LEBSANFT, Franz (2005): «Kommunikationsprinzipien, Texttraditionen, Geschichte», en Schrott, Angela y Harald Völker, coord., Historische Pragmatik und historische Varietätenlinguistik in den romanischen Sprachen, Göttingen, Göttinger Universitätsverlag, 25-44. 
LEBSANFT, Franz (2015): «Aktualität, Individualität und Geschichtlichkeit. Zur Diskussion um den theoretischen Status von Diskurstraditionen und Diskursgemeinschaften", en Lebsanft, Franz y Angela Schrott, coord., Diskurse, Texte, Traditionen. Methoden, Modelle und Fachkulturen in der Diskussion, Bonn, Bonn University Press, Vandenhoeck \& Ruprecht, 97114.

LeECH, Geoffrey (1983): Principles of Pragmatics, New York/London, Longman.

LEMPERT, Michael (2012): «Indirectness», en Bratt Paulston, Christina et alii, coord., The Handbook of Intercultural Discourse and Communication, Oxford, Blackwell, 180-204.

MÁrquez ReITER, Rosina (2000): Linguistic Politeness in Britain and Uruguay: A Contrastive Study of Requests and Apologies, Amsterdam/Philadelphia, Benjamins.

MeIBAUER, Jörg (1986): Rhetorische Fragen, Tübingen, Niemeyer.

MIHATSCH, Wiltrud (2010): «Wird man von Hustensaft wie so ne art bekifft?» Approximationsmaerker in romanischen Sprachen, Frankfurt am Main, Klostermann.

Monjour, Alf (2006): «Pasad, pasad-Kommen Sie bitte 'rein. Pedro Almodóvar, los actos de habla y la comparación intercultural», en SchraderKniffki, Martina, coord., La cortesía en el mundo hispánico. Nuevos contextos, nuevos enfoques metodológicos, Frankfurt am Main, Vervuert, 1542.

MULDER, Gijs (2003): "¿Por qué no coges el teléfono?», en Haverkate, Henk et alii, coord., Aproximaciones pragmalingüísticas al español, Amsterdam, Rodopi, 181-207.

MULKEN, Margo van (1996): «Politeness Markers in French and Dutch Requests", Language Sciences, 1, 698-702.

OESTERREICHER, Wulf (2001): «Historizität Sprachvariation, Sprachverschiedenheit, Sprachwandel», en Haspelmath, Martin et alii, coord., Sprachtypologie und sprachliche Universalien. Ein internationales Handbuch, tomo 2, vol. 2, Berlin/New York, De Gruyter, 15541595.

OGIERMANN, Eva (2012): «About Polish Politeness», en Ruiz de Zarobe, Leyre y Yolanda Ruiz de Zarobe, coord., Speech Acts and Politeness across Languages and Cultures, Bern y otras, Peter Lang, 27-52.
Placencia, Maria E. y Diana Bravo, coord. (2002): Actos de habla y cortesía en español, München, Lincom.

Placencia, María E. y Carmen García (2007a): «Preface», en Placencia, María E. y Carmen García, coord., Research on Politeness in the Spanish Speaking World, London, Erlbaum, XIII$\mathrm{XVII.}$

Placencia, María E. y Carmen García (2007b): "Salient Trends and Directions for Future Research", en Placencia, María E. y Carmen García, coord., Research on Politeness in the Spanish Speaking World, London, Erlbaum, 369383.

PlaCENCIA, María E. y Carmen GaRCíA, coord., (2007): Research on Politeness in the Spanish Speaking World, London, Erlbaum.

SCHLIEBEN-LANGE, Brigitte (1983): Traditionen des Sprechens. Elemente einer pragmatischen Sprachgeschchtsschreibung, Stuttgart y otras, Kohlhammer.

SCHRADER-KNIFFKI, Martina, coord. (2006): La cortesía en el mundo hispánico. Nuevos contextos, nuevos enfoques metodológicos, Frankfurt am Main, Vervuert.

SCHROTT, Angela (2014): «Sprachwissenschaft als Kulturwissenschaft aus romanistischer Sicht: Das Beispiel der kontrastiven Pragmatik», en: Romanische Forschungen 126 , 3-44.

SCHROTT, Angela (2015): «Kategorien diskurstraditionellen Wissens als Grundlage einer kulturbezogenen Sprachwissenschaft», en: Lebsanft, Franz y Angela Schrott, coord., Diskurse, Texte, Traditionen. Modelle und Fachkulturen in der Diskussion, Bonn/Göttingen, Vandenhoeck \& Ruprecht/Bonn University Press, 115-146.

SEARLE, John R. (1969): Speech Acts. An Essay in the Philosophy of Language, Cambridge, Cambridge University Press.

SEARLE, John R. (1979a): «A Taxonomy of Illocutionary Acts", en Searle, John R., Expression and Meaning. Studies in the Theory of Speech Acts, Cambridge, Cambridge University Press, 1-29.

SEARLE, John R. (1979b): «Indirect Speech Acts», en Searle, John R., Expression and Meaning. Studies in the Theory of Speech Acts, Cambridge, Cambridge University Press, 30-57.

SIEBOLD, Kathrin (2008): Actos de habla y cortesía verbal en español y en alemán. Estudio 
pragmalingüístico e intercultural, Frankfurt am Main y otras, Peter Lang.

TrOsBORG, Anna (2010): «Introduction», en Trosborg, Anna, coord., Pragmatics across Languages and Cultures, Berlin/New York, De Gruyter Mouton, 139.

VERSCHUEREN, Jef (1995): «The Pragmatic Perspective", en Verschueren, Jef, coord., Handbook of Pragmatics, Amsterdam/Philadelphia, Benjamins, 1-19.

VERSCHUEREN, Jef (2009): «Introduction: The pragmatic perspective», en Verschueren, Jef y Jan-Ola Östman, coord., Key Notions for Pragmatics, Amsterdam/Philadelphia, 1-27.

WALDENFELS, Bernhard (1994): Antwortregister, Frankfurt am Main, Suhrkamp.

WIERZBICKA, Anna (2003): Cross-Cultural Pragmatics. The Semantics of Human Interaction, Berlin/New York, De Gruyter, 2. coord.

WieRZBICKA, Anna (2010): "Cultural Scripts and Intercultural Communication», en: Trosborg, Anna, coord., Pragmatics across Languages and Cultures, Berlin/New York, De Gruyter, 43-78 
Cortesía verbal y competencia lingüística...| Angela Schrott 